\title{
Editorial
}

\section{Nonlinear Dynamics in Financial Systems: Advances and Perspectives}

\author{
Chuangxia Huang, ${ }^{1}$ Fenghua Wen, ${ }^{2}$ Jianping Li, ${ }^{3}$ Taishan Yi, ${ }^{4}$ and Xiaodong Lin ${ }^{5}$ \\ ${ }^{1}$ College of Mathematics and Computing Science, Changsha University of Science and Technology, Changsha 410114, China \\ ${ }^{2}$ Business School, Central South University, Changsha 410012, China \\ ${ }^{3}$ Institute of Policy and Management, Chinese Academy of Science (CAS), Beijing 100190, China \\ ${ }^{4}$ Department of Applied Mathematics, University of Western Ontario, London, ON, Canada N6A 5B7 \\ ${ }^{5}$ Rutgers Business School, Rutgers University, Piscataway, NJ 08854, USA
}

Correspondence should be addressed to Chuangxia Huang; cxiahuang@126.com

Received 18 December 2014; Accepted 18 December 2014; Published 31 December 2014

Copyright (C) 2014 Chuangxia Huang et al. This is an open access article distributed under the Creative Commons Attribution License, which permits unrestricted use, distribution, and reproduction in any medium, provided the original work is properly cited.

In the recent years, nonlinear dynamics have attracted rapidly growing attention in the fields of finance, economy, mathematical biology, and many other disciplines. In spite of the amount of published results recently focused on financial systems, there remain many challenging open questions. It is important to develop new theories and methods, as well as to modify and refine the well-known techniques for the analysis of new classes of problems.

The aim of this special issue is to gather recent research efforts on the development and applications of nonlinear dynamics. This special issue contains thirty-five research articles. The original papers explored in this special issue include a wide variety of topics such as the following.

Behavioral Finance Modeling. Z. Liu et al. proposed a new model of sentiment contagion, named the SOSa-SPSa model, and found that both numbers of optimism and pessimism will increase with the probability of spontaneity or contagion. $\mathrm{X}$. Gong et al. divided the logarithmic realized volatility into the logarithmic continuous sample path variation and the logarithmic discontinuous jump variation on the basis of the SV-RV model.

J. Jin et al. analysed the impact of the fluctuation of the international gold prices on the Chinese gold stocks. X. Wang et al. investigated the relations between Qualified Foreign Institutional Investors (QFII) holdings and the performance of the A-share listed companies. W. Ye et al. investigated measuring contagion of subprime crisis based on MVMQCAViaR method. C. Huang et al. constructed a new proxy for information asymmetry based on the principal component analysis.

Risk Assessment and Credit Analysis. P. Li and J. Song investigated the pricing Chinese convertible bonds with dynamic credit risk. G. Xing et al. proposed a dynamic multiple setting model for gap risk management. G. Chen et al. utilized the realized jump components to explore a new jump (including nonsystematic jump and systematic jump) risk factor model.

R. Chen and H. Yu developed a mixed Poisson model assuming that default probabilities of obligors depend on a set of common economic factors to construct the dependence structure of obligors. L. Yu proposed a least squares fuzzy support vector machine (LS-FSVM) model for the purpose of credit risk evaluation. F. Wen et al. showed that investors' risk preference is time varying and is influenced by previous outcomes.

Portfolio Selection and Optimization. J. Huang et al. showed that incorporating overconfidence into real option decisionmaking model of metal mineral resources development is a crucial extension of project evaluation theory. W. Bi et al. studied the dynamic portfolio choice problem with multiple 
variables of stochastic volatility and agents' limited attention. M. Zhong et al. investigated project capital allocation combination equilibrium decision model based on behavioral option game.

Asset Pricing and Arbitrage Techniques. G. Bi et al. constructed a dynamic game to build a two-period dynamic pricing model for two brands of substitutes which are sold by duopoly. J. Liu et al. showed that the proposed LSRQM model fits well the market prices of convertible bonds in China's market and the LSRQM method is effective. Y. Liu et al. rebuilt the linear demand function considering free-riding behavior and modified the pricing model based on channel fairness.

Dynamical Analysis of Stability, Chaos, and Bifurcation on Financial Systems. L. Gori et al. showed that nonlinear dynamic phenomena, such as coexistence of attractors and local and global bifurcations, occur due to the existence of a time gap in the process of adjustment of market prices. J. Luo et al. investigated linear control of fractional-order financial chaotic systems with input saturation. H. Wang et al. investigated the dynamics of third order nonlinear neutral equations.

Numerical Computation and Simulations. Q. Wu and H. Xiao proposed a dynamic CGE model and the simulation results show that changes in supply will affect the labor market structure, the relative factor price, and the investment in and the output of industries. G.-J. Wang et al. proposed a timevarying correlation network-based approach to investigate dynamics of foreign exchange (FX) networks.

Financial Diagnosis and Control. X. Wang et al. adopted propensity score matching (PSM) method to conduct empirical analysis about the treatment effects of indirect financing level of SMEs under different systems.

The response to this special issue was beyond our expectation. We received 61 papers in the interdisciplinary research fields. This special issue includes thirty-five highquality peer-reviewed articles. These articles contain several new, novel, and innovative techniques and ideas that may stimulate further research in every branch of pure and applied sciences.

\section{Acknowledgments}

We would like to express our deepest gratitude to the reviewers, whose professional comments and valuable suggestions guaranteed the high quality of these selected papers. We would like to express our gratitude to the authors for their interesting and novel contributions. We would also like to thank the editorial board's members of this journal, for their support and help throughout the preparation of this special issue. The interested readers are advised to explore these interesting and fascinating fields further. We hope that problems discussed and investigated in this issue may be inspiration and motivation to discover new, innovative, and novel applications in all areas of nonlinear financial dynamics. Chuangxia Huang would like to express the gratitude to the support of the National Natural Science Foundation of China (no. 11101053 and no. 71471020), the Key Project of the Chinese Ministry of Education (no. 211118), and the China Postdoctoral Science Foundation (no. 20140550097).

Chuangxia Huang Fenghua Wen Jianping $\mathrm{Li}$ Taishan Yi Xiaodong Lin 


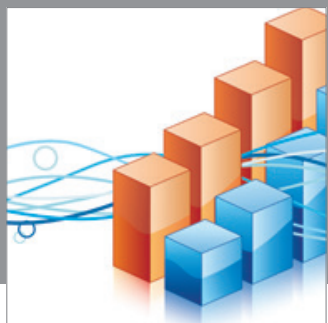

Advances in

Operations Research

mansans

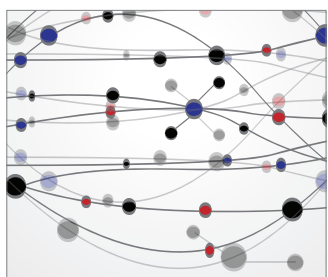

The Scientific World Journal
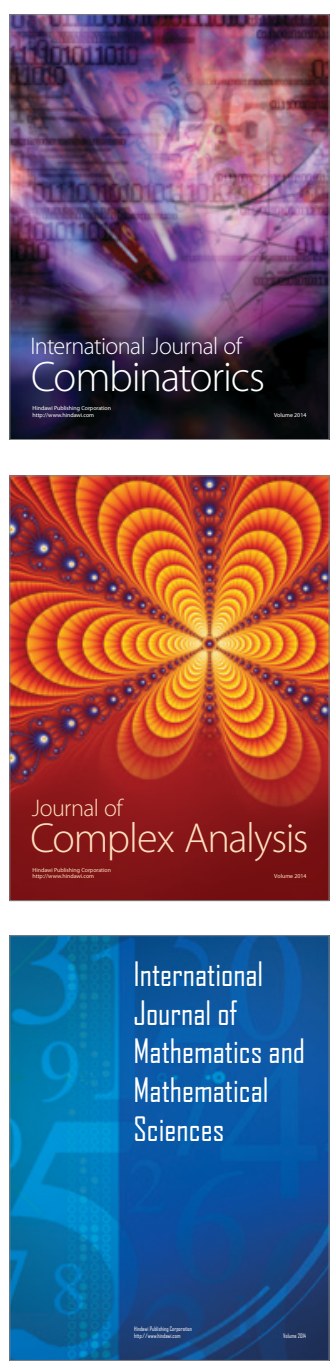
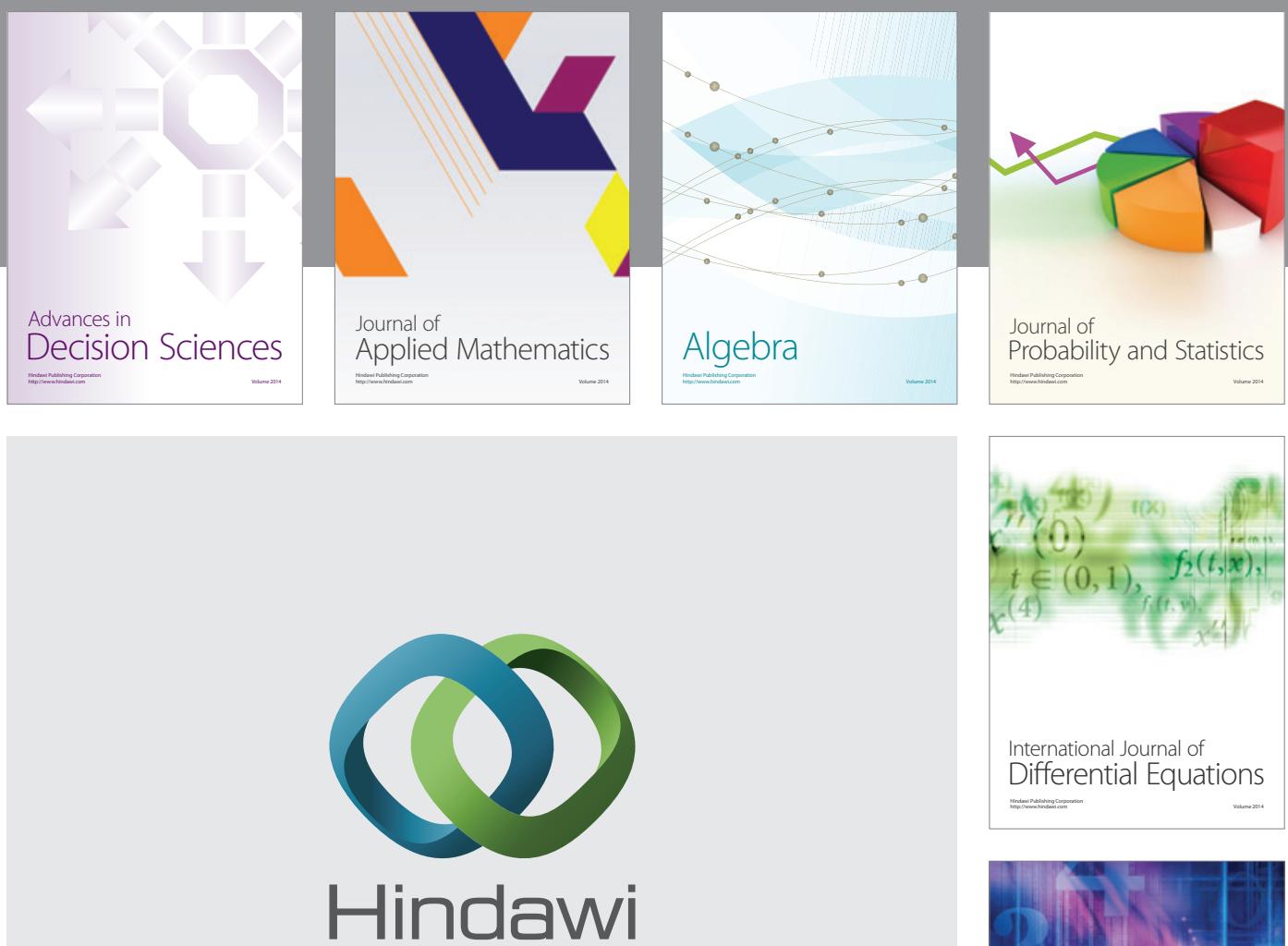

Submit your manuscripts at http://www.hindawi.com
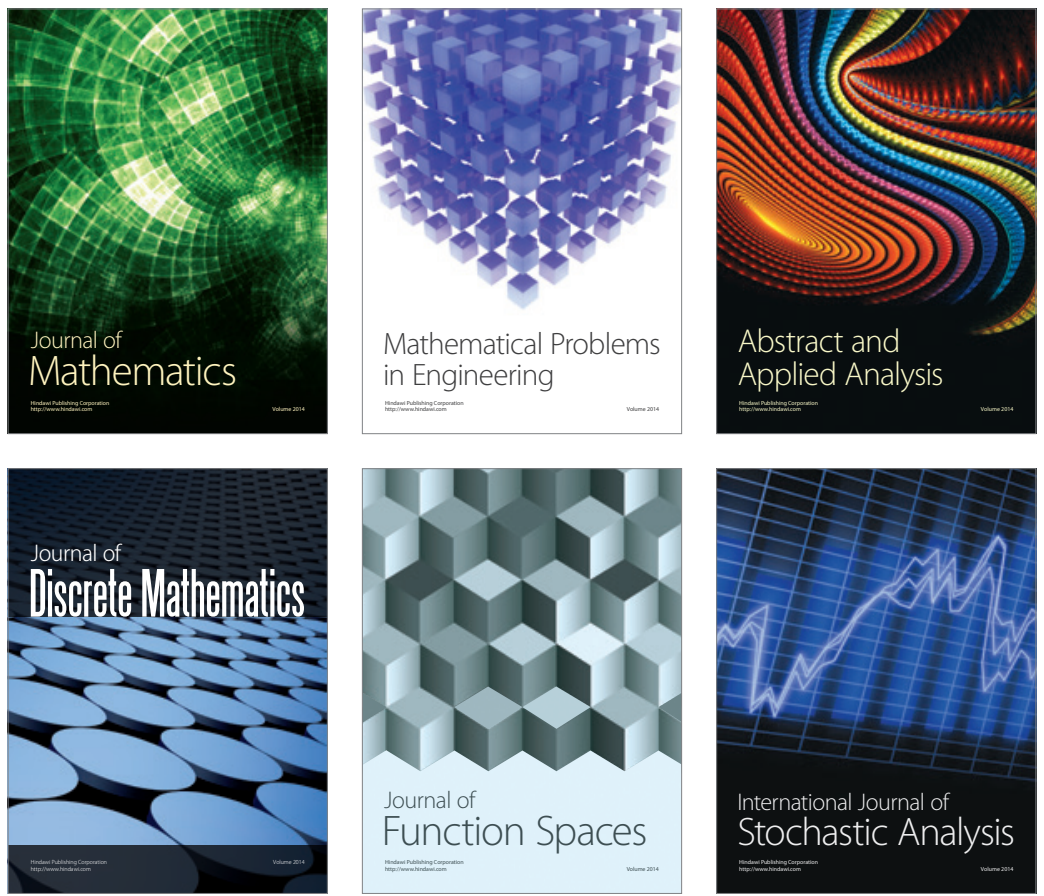

Journal of

Function Spaces

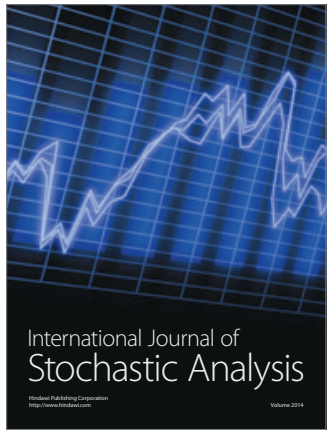

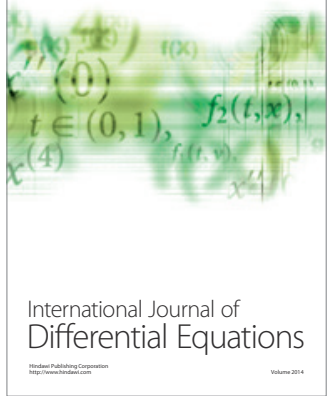
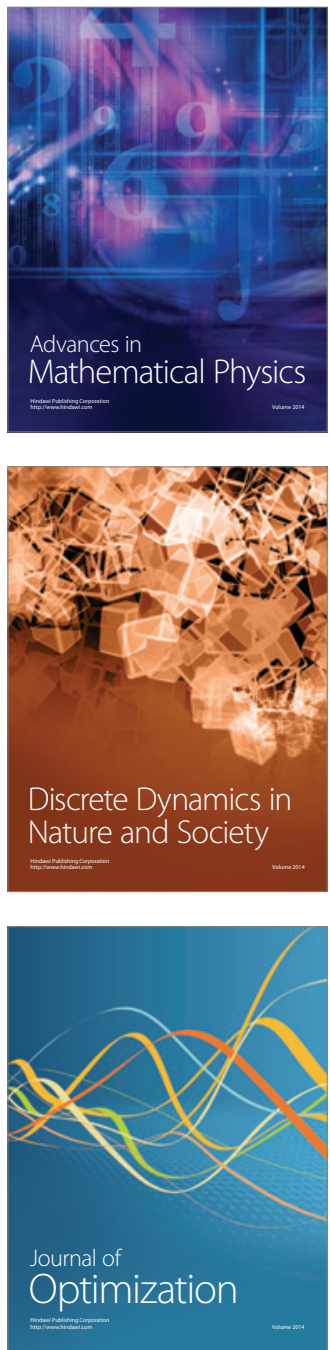